
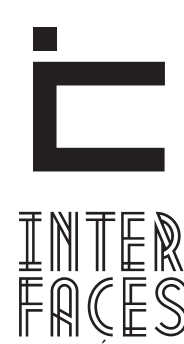

CIENTÍFICAS

HUMANAS E SOCIAIS

ISSN IMPRESSO 2316-3348

E-ISSN 2316-3801

DOI - 10.17564/2316-3801.2017v6n1p47-58

\title{
INFLUÊNCIA DA MASCULINIDADE NAS CONCEPÇÕES E PRÁTICAS DE SAÚDE-DOENÇA DE ALUNOS DA EDUCAÇ̃̃O SUPERIOR EM SAÚDE
}

\author{
INFLUENCE OF THE MALE IN THE CONCEPTIONS AND PRACTICES OF HEALTH-DISEASE OF STUDENTS OF HIGHER EDUCATION IN HEALTH \\ INFLUENCIA DELLAMASCULINIDADENLASCONCEPCIONESY PRÁCTICAS DE SALUD-ENFERMEDAD DE ALUMNOS DELA EDUCACIÓN SUPERIOR EN SALUD
}

Maria Thereza Ávila Dantas Coelho ${ }^{1}$

Daniele Machado Pereira Rocha²

Raquel Araújo da Silva Carneiro ${ }^{3}$

\section{RESUMO}

As concepções e práticas relacionadas à saúde e doença estão ligadas às conjunturas sociais, econômicas, políticas e culturais de cada indivíduo e englobam aspectos individuais, subjetivos, multidimensionais e intersetoriais. 0 padrão hegemônico de masculinidade distanciou os homens do objeto saúde, contribuindo para a falta de cuidado e escassez de pesquisas, envolvendo esses sujeitos. Objetivo: analisar a influência da masculinidade nas concepções e práticas de estudantes de um curso superior em saúde, relacionadas à saúde e à doença. Método: participaram desta pesquisa 76 discentes homens, que responderam a um questionário semiestruturado. As respostas foram interpretadas nos moldes da análise de conteúdo de Bardin. Resultados: concepções de saúde associadas às dimensões físicas e psicológicas e à qualidade de vida foram prevalentes. A doença foi relacionada às ideias de desequilíbrio e alteração da normalidade. Concepções de saúde como ausência de doença e vice-versa foram minoritárias. Dentre as ações para manter a saúde e prevenir doenças, destacam-se a atividade física e a alimentação saudável. Quando doentes, 93\% dos participantes afirmaram buscar um profissional da saúde e $71 \%$ praticar a automedicação, enquanto as práticas alternativas e/ou complementares foram relatadas em menor proporção (32\%), bem como as religiosas (20\%). Conclusão: nota-se que entre os discentes o binômio saúde-doença é percebido como fenômeno complexo e multifatorial. Majoritariamente há preocupação com hábitos 
preventivos e de vida saudável - características de cuidado e noções de saúde que se distanciam do modelo hegemônico de masculinidade e saúde discutido entre pesquisadores(as) desta temática.

\section{ABSTRACT}

The conceptions and practices related to health and disease are linked to social, economic, political and cultural conjunctures of each individual and include individual, subjective, multidimensional and intersectoral aspects. The hegemonic patter of masculinity distanced men from the health object, contributing to the lack of care and scarcity of research involving these individuals. This article has as objective to analyze the influence of masculinity in the conceptions and practices of students of higher education in health related to health and disease. The method used in this work says about seventy-six male students participated in this research, answering a semi-structured questionnaire. The answers were interpreted along the lines of Bardin's content analysis. As results, we have that health's conceptions associated with physical and psychological dimensions and with quality of life were prevalent. The disease was related to the ideas of imbalance and altered normality. Concep-

\section{RESUMEN}

Las concepciones y prácticas relacionadas con salud y enfermedad están conectadas a coyunturas sociales, económicas, políticas y culturales de cada individuo e incluyen aspectos individuales, subjetivos, multidimensionales e intersectoriales. El modelo hegemónico de masculinidad distanció los hombres del objeto salud, contribuyendo para la falta de cuidado y escasez de investigaciones acerca de esos individuos. Objetivo: analizar la influencia de la masculinidad en las concepciones y prácticas de los estudiantes de un

\section{PALAVRAS-CHAVE}

Saúde. Masculinidade. Educação Superior.

tions of health as absence of disease and vice versa were minority. Among the actions for maintain health and prevent diseases, physical activity and healthy eating were highlighted. When sick, $93 \%$ of participants affirmed seeking a health professional and $71 \%$ practiced self-medication, while alternative and / or complementary practices were reported to a lesser extent (32\%), as well the religious ones (20\%). It is noticed that among the students the health-disease binomial is perceived as a complex and multifactorial phenomenon. There is a major concern with preventive and healthy life habits characteristics of care and health notions that distance themselves from the hegemonic model of masculinity and health discussed among researchers of this theme.

\section{KEYWORDS}

Health. Masculinity. Higher education.

grado en salud relacionadas con salud y enfermedad. Método: participaron en esta investigación 76 estudiantes hombres, que respondieron a un cuestionario semiestructurado. Las respuestas fueron interpretadas en los moldes del análisis de contenido de Bardin. Resultados: concepciones de salud asociadas con las dimensiones físicas y psicológicas y la calidad de vida predominaron. La enfermedad fue relacionada con desequilibrio y alteración de la normalidad. Concepciones de salud como ausencia de enfermedad y vice- 
versa fueron minoría. Entre las acciones para mantener la salud y prevenir la enfermedad, se destacan la actividad física y la alimentación saludable. Cuando enfermos, 93\% de los participantes dijeron que buscan un profesional de la salud y $71 \%$ practicar la automedicación; las prácticas alternativas y/o complementarias se han reportado en menor medida (32\%), así como las religiosas (20\%). Conclusión: se observa que entre los estudiantes el binomio salud-enfermedad es percibido como fenómeno complejo y multifac- torial. Predominantemente existe una preocupación con hábitos de prevención y de vida saludable - características de cuidado y nociones de salud que se distancian del modelo hegemónico de masculinidad y salud discutido entre los investigadores.

\section{PALABRAS CLAVE}

Salud. Masculinidad. Educación superior.

\section{INTRODUÇ̄̃̃O}

O processo saúde-doença, durante a história, apresentou grande variabilidade de definição. Scliar (2007) aponta que o conceito de saúde é reflexo das conjunturas sociais, econômicas, políticas e culturais, de forma que a saúde não pode representar a mesma coisa para todas as pessoas, assim como "aquilo que é considerado doença varia muito” (SCLIAR, 2007, p. 30). Batistella (2007), por sua vez, assinala que o conceito de saúde não recebia o mesmo destaque que o de doença e que, graças à hegemonia do modelo biomédico $^{1}$, a saúde foi internalizada, pela população, como sendo somente a ausência de doença.

Uma das proposições de conceito universal de saúde, que contraria a perspectiva biomédica, foi realizada após o fim da segunda Guerra mundial, quando foram criadas a Organização das Nações Unidas (ONU) e a Organização Mundial da Saúde (OMS), que definiu, na sua carta de princípios de 7 de abril de 1948, que "saúde é o estado do mais completo bem-estar físico, mental e social e não apenas a ausência de enfermidade", além de reconhecer a obrigatoriedade do Esta-

1 "Caracterizado pela ênfase nos aspectos biológicos, individuais e pela abordagem mecanicista, esse modelo passa a fragmentar o corpo em sistemas, órgãos, tecidos e células, estruturando um conhecimento cada vez mais especializado sobre cada função e disfunção orgânica" (BATISTELLA, 2007, p. 54). do na promoção e proteção da saúde (SCLIAR, 2007, p. 37). Para Scliar (2007), os valores e contextos individuais são determinantes do processo saúde-doença (época, lugar, classe social, concepções científicas, religiosas e filosóficas), logo é fundamental que, além das fundamentações teóricas, as concepções de saúde englobem também aspectos individuais, subjetivos, multidimensionais e intersetoriais.

Quanto ao conceito de doença, Czeresnia (2009) afirma que este foi constituído mediante a redução do corpo humano, pensado a partir de constantes morfológicas e funcionais. A autora considera ainda que existe uma distância entre o conceito de doença, enquanto construção mental, e o adoecer, enquanto experiência de vida. Nesse sentido, Boruchovitch e outros autores (1991) afirmam que o conceito de doença não se limita a um distúrbio da saúde e ao contexto biológico do indivíduo, centrado na corpo e na mente, mas avança na direção social e histórica do mesmo. Sendo assim, a experiência do adoecimento está relacionada a uma série de fatores, como aponta Czeresnia (2009, p. 46): "a saúde e o adoecer são formas pelas quais a vida se manifesta" e correspondem a experiências singulares e subjetivas.

No campo que integra a subjetividade e os aspectos biológicos também residem as práticas de saúde, 
definidas por auxiliarem no enfrentamento de doenças, relacionadas a aspectos educacionais, familiares e culturais, bem como ao autocuidado, motivações individuais, com livre escolha de equipamentos e materiais por parte do indivíduo, com o intuito de preservar a sua vida e saúde (OREM, 2001 apud SILVA et al., 2009). Corroborando com o autor acima, Teixeira e Vilasboas (2013) compreendem que as práticas de saúde promovem a melhoria das condições de vida, reduzem os riscos e danos à saúde e reabilitam os indivíduos, podendo ser executadas na residência, escola, trabalho e serviços de saúde.

Carvalho e Luz (2009) também consideram que as práticas de saúde, compreendidas como ações coletivas e/ou individuais que auxiliem no processo de cura e na preservação da saúde, têm diversos planos e possibilidades, já que são um espaço de objetificação e articulação de elementos simbólicos. Assim, a escolha das mesmas tem um caráter subjetivo e relacional. Nesse sentido, Ayres (2001) discute o conceito de (inter)subjetividade, considerando "o caráter imediatamente relacional e irremediavelmente contingente de nossas identidades e historicidades como indivíduos e grupos" (AYRES, 2001, p. 65). Desse modo, as (inter) subjetividades são caracterizadas pela mobilidade do sucesso prático (AYRES, 2001), dependendo do indivíduo, mas de modo transformador, pois seus principais determinantes seriam aspectos subjetivos e pessoais.

Seguindo o pensamento de autores citados acima, as concepções e práticas ligadas à saúde e à doença são permeadas pelo modelo explicativo dos Determinantes Sociais da Saúde (DSS) que, de acordo com a Comissão Nacional sobre os Determinantes Sociais da Saúde (CNDSS), compreende os "fatores sociais, econômicos, culturais, étnico-raciais, psicológicos e comportamentais que influenciam a ocorrência de problemas de saúde e seus fatores de risco na população" (BUSS; PELLEGRINI FILHO II, 2007, p. 78), afastando dos sujeitos a culpabilização completa pelo seu estado de saúde.

No tocante às concepções e práticas dos homens relacionadas à saúde e à doença, Gomes (2010) compreende que os estereótipos sociais e a construção de gênero, que dão significado à ideia de masculinida- de enquanto um conjunto de características, valores e condutas esperadas de um homem, influenciam os hábitos relacionados à saúde, deste segmento populacional, produzindo um distanciamento dos cuidados e diálogos acerca da saúde.

Figueiredo (2005) considera que, no modelo de masculinidade idealizada, estão presentes as noções de invulnerabilidade e comportamento de risco, o que dificulta a expressão pelos homens das próprias necessidades de saúde. A construção da identidade masculina visa se opor às ideias de fraqueza e feminilidade, de modo que a forma como os homens usam e percebem seus corpos interfere nas suas condições de saúde, uma vez que estes desvalorizam o autocuidado e têm preocupação incipiente com a própria saúde.

A perspectiva de Figueiredo (2005) corrobora a ideia de Connell (1995), segundo a qual a masculinidade hegemônica - conjunto de práticas associadas como padrão para os homens - produz um déficit na saúde do homem, por fazer emergir importantes fatores de risco para o adoecimento. Connell (1995) considera que a masculinidade hegemônica, além de ser uma expectativa de papeis ou identidades, é entendida também como um padrão de práticas que, mesmo se apenas uma minoria dos homens a adota, foi instituída como norma. Em uma sociedade tipicamente patriarcal como a brasileira, as cobranças relacionadas ao modelo de masculinidade hegemônica são inerentes ao cotidiano dos homens.

Entretanto, com os diversos avanços sociais, atualmente a dicotomia e os padrões masculino/feminino já não enquadram todas as pessoas como antes. A crise da masculinidade atual seria assim o reflexo da existência de múltiplas masculinidades, em detrimento da hegemônica. É importante distinguir a masculinidade como princípio simbólico e as várias masculinidades no sentido de identidades masculinas, de caráter plural e dinâmico (SCHRAIBER; GOMES; COUTO, 2005).

Faz-se necessário, assim, entender as construções sociais das masculinidades para, então, analisar as concepções e práticas dos homens ligadas à saúde e à doença. Nesse contexto, a proposta deste artigo é analisar as concepções e práticas dos estudantes do 
gênero masculino ingressos em 2014 no Bacharelado Interdisciplinar em Saúde (BIS) da Universidade Federal da Bahia (UFBA), ligadas à saúde e à doença, considerando as suas relações com a(s) masculinidade(s).

\section{METODOLOGIA}

Este artigo é fruto de um projeto de pesquisa intitulado Concepções e práticas pessoais e profissionais ligadas a processos de saúde e doença. Para a realização desta pesquisa adotou-se um questionário semiestruturado, no qual constavam 33 perguntas relacionadas às concepções e práticas de saúde. Neste artigo são discutidas as respostas dos estudantes do gênero masculino, ingressos em 2014 no Bacharelado Interdisciplinar em Saúde da Universidade Federal da Bahia. Os discentes frequentavam o curso nos turnos vespertino e noturno e tinham idades variantes entre 18 e 61 anos.

Do questionário, foram selecionadas aqui sete perguntas concernentes às concepções e práticas ligadas à saúde e doença dos participantes: 1. "O que é saúde para você”? 2. "Quais dessas ações você realiza para manter a sua saúde"? 3. "O que é doença para você"? 4. "Quais dessas ações você já realizou para prevenir doenças"? 5. "O que você faz quando está doente"? 6. "Em geral, faz uso de medicamentos"? 7. "Qual(is) são as práticas integrativas/complementares e/ou religiosas que você utiliza"? 0 total de participantes do gênero masculino foi de 76 alunos, que responderam ou não às perguntas supracitadas, de forma livre e sem identificação.

Dentre as perguntas selecionadas, algumas eram de natureza descritiva e outras eram objetivas. Por conseguinte, a análise e interpretação dos dados foram realizadas de duas formas distintas: as respostas das perguntas 1 a 4, que eram abertas, foram digitadas no processador de texto Microsoft Word® e analisadas de forma qualitativa, visando explorar o caráter subjetivo de cada resposta e interpretar seu conteúdo dentro de um quadro de referência. As perguntas 5 a 7 , que eram objetivas, foram contabilizadas em número absoluto de estudantes e porcentagem, a partir do editor de plani-
Ihas Microsoft Office Exce/®, e analisadas de forma quantitativa, a fim de se observar padrões reconhecíveis e mensurá-los. Essas perguntas apresentavam a possibilidade de se assinalar um ou mais itens.

Este estudo é, portanto, qualitativo e quantitativo. Os seus dados foram analisados com base na análise de conteúdo, com foco na proposta de Bardin (2009) da análise temática, definida como um conjunto de técnicas de análise que utiliza procedimentos sistemáticos e objetivos de descrição do conteúdo, a fim de produzir sentidos e significados. No presente estudo, as respostas dos sujeitos foram interpretadas à luz da revisão de literatura.

Ressalta-se que todas as questões foram respondidas de forma livre. Nas questões dissertativas, os estudantes tinham a oportunidade de escrever sobre o tema sem a presença de respostas preestabelecidas. Até mesmo as perguntas objetivas continham a opção "outros", para que os estudantes pudessem apresentar respostas mais condizentes com a sua realidade, que não estavam presentes entre as opções. Algumas questões contavam ainda com uma variação de múltipla escolha.

Este estudo foi aprovado pelo Comitê de Ética em Pesquisa (CEP) da Escola de Enfermagem da UFBA, sob o parecer $n^{0} 741.187$, respeitando os dispositivos da Resolução n ${ }^{0}$ 466/13 do Conselho Nacional de Saúde sobre Pesquisa com Seres Humanos. Os estudantes participantes assinaram um termo de consentimento livre e esclarecido (TCLE).

\section{RESULTADOS E DISCUSSÃO}

A partir da análise das respostas dos estudantes, buscou-se identificar as suas concepções e práticas de saúde e doença. Acerca das concepções, os alunos, quando questionados sobre o que é saúde, responderam que corresponde a um completo estado de bem-estar, muitas vezes associado às dimensões física e psicológica; esta resposta equivale a $37 \%$ (28 respostas) do total de 76 . Há uma presença de respostas relativas à saúde em seu conceito ampliado, associando-a a qualidade de vida e ao meio; estas foram 
apresentadas por $29 \%$ dos estudantes (22 sujeitos), conforme algumas exemplificações abaixo:

É um conceito amplo que percorre a dimensão econômica, social, cultural, histórica, psicológica, ambiental e biológica do ser.

Saúde é o bem-estar físico, social, político e cultural, ou seja, é ter uma boa educação, um renda adequada, lazer, esporte, entretenimento. Não somente a ausência de enfermidades, embora seja um componente importante.

Observa-se que esses estudantes destacaram a complexidade do objeto saúde, ao evidenciar que a saúde não está relacionada apenas com a ausência de doença. Além de apresentarem um conceito ampliado de saúde, os discentes apontaram sua relação com a ideia de qualidade de vida socialmente determinada, associada a dimensões psicológicas e aos determinantes sociais da saúde (DSS), como cultura, política, lazer, renda, dentre outros (BUSS; PELLEGRINI FILHO II, 2007). Apenas 4\% (3 discentes) revelaram a concepção restrita de saúde como ausência de doença.

De forma menos prevalente, alguns alunos conceituaram a saúde como um estado de harmonia e/ou equilíbrio entre as partes, o que correspondeu a $20 \%$ das respostas (15 estudantes), conforme os exemplos abaixo:

Saúde para mim é a junção dos meus poderes e deveres físicos, espirituais, sociais e econômicos. Onde há harmonia entre estes e mais do que isto, onde todos estes fatores estão em plena funcionalidade e em níveis aceitáveis.

É a harmonia da mente e corpo do ser vivo em questão. Sendo assim saudável quando estes dois estão em equilíbrio.

Estado físico e mental estável, em equilíbrio, sem a necessidade do uso de medicamentos para a sua manutenção.

Um aluno declarou que a saúde é um "conceito muito relativo. Não dá para definir". Outro disse que ela é "estar bem consigo mesmo, ter uma boa qualidade de vida, ter um equilíbrio mental". Essas respostas reiteram que os estudantes do BIS/UFBA percebem a saúde como um fenômeno complexo, relacionado a diversos fatores. 0 estudo de Nascimento e outros autores (2011) sobre masculinidades e práticas de saúde aponta dados semelhantes às respostas dos alunos do BIS/UFBA, já que nesse estudo a representação social da saúde entre os entrevistados apresenta como elementos centrais os marcadores "bem-estar", "cuidado", "importante".

Quanto ao conceito de doença, as respostas dos estudantes foram predominantemente ligadas ao desequilíbrio/desarmonia (39\%, 30 respostas) e, de forma mais moderada, ao mal-estar (11\%, 8 respostas). A predominância de uma definição não restrita à dimensão biológica da doença permite supor que a maioria dos discentes compreende a complexidade do processo saúde-doença, ou já não se restringe à compreensão da doença como simplesmente uma patologia orgânica. Os exemplos abaixo apresentam algumas respostas dessa natureza:

É um estado patológico, onde há desarmonia entre os sistemas, seja físico ou mental.

Doença é tudo que ameaça o complexo da saúde, ou seja, "quebrando" a sua harmonia psicológico-físico.

Algo que prejudique o bem-estar de um indivíduo, podendo ser de ordem epidemiológica ou até mesmo de caráter psicológico.

Houve também respostas relacionadas à alteração da normalidade (21\%, 16 respostas) e, de forma menos frequente, à doença como um estado de ausência de saúde (9\%, 7 respostas). A partir da análise desses últimos dados, pode-se inferir que alguns discentes reproduzem a concepção de doença que integra o senso comum. No entanto, as respostas que relacionaram a doença a palavras como "dor", "desconforto" e "incômodo" tiveram uma frequência muito menor - apenas 4\% (3 respostas) -, a exemplo da resposta do estudante que declarou: "Uma patologia, fruto de maus cuidados, dor, aflição, infelicidade”. Os exemplos abaixo sintetizam as respostas relacionadas à alteração da normalidade e ausência de saúde: 
É quando seu corpo ou sua mente sai dos padrões normais. É a vulnerabilidade no qual o corpo passa de seu estado normal para anormal.

Condição inversa à saúde.

Mesmo que alguns tenham uma concepção mais restrita da doença, as concepções dos estudantes demonstram, predominantemente, que estes já compreendem a dinamicidade dos processos de adoecimento e a complexidade do processo saúde-doença. No estudo de Camargo e outros autores (2011, p. 189), acerca das representações sociais de saúde e cuidado, com homens de todas as faixas etárias, residentes em Goiânia e Florianópolis, os autores concluem que "[...] a maioria dos homens considera que uma alimentação saudável e a prática regular de atividade física constituem cuidados importantes para a saúde", o que demonstra que as concepções desse segmento da população sobre cuidados com a saúde estão sendo alteradas lentamente.

Para esses autores, a vulnerabilidade dos homens à doença é caracterizada pela incipiente perspectiva da prevenção. Os dados dessa pesquisa se assemeIham com as respostas dos discentes do BIS/UFBA, tendo em vista que as concepções de saúde apresentadas aqui se distanciam do imaginário de que os homens não se preocupam com a própria saúde.

Nessa perspectiva, em estudo sobre representações sociais de homens acerca dos processos saúde-doença, Silva (2013, p.46) aponta que “[...] a saúde é considerada uma realização individual do sujeito à medida que este adquire hábitos que favoreçam as condições de vida e bem-estar". A autora considera a saúde como uma realização individual, pois os entrevistados evidenciam a relação entre hábitos saudáveis e equilíbrio emocional, incluindo atividade física, alimentação saudável, preservação do sono e acompanhamento médico como ações para alcançar a saúde (SILVA, 2013).

Silva (2013, p. 46) aponta ainda que, em sua pesquisa, "a saúde é compreendida de uma forma mais ampla, envolvendo questões políticas, econômicas, sociais, culturais, ambientais e biológicas”. A percepção e as ações dos participantes do presente estudo corro- boram as considerações de Silva (2013), já que as respostas dos alunos apresentam, predominantemente, concepções e práticas de saúde ampliadas e complexas, assim como a dinâmica do binômio saúde-doença.

Sobre as ações realizadas para preservar a saúde e prevenir doenças, as respostas se assemelharam em quase todos os aspectos. As ações com maior destaque, em praticamente todas as respostas, foram as da ida regular ao médico (93\%, 71 alunos), prática de atividade física, (84\%, 64 estudantes) e preocupação com uma alimentação saudável ( $72 \%$, 55 discentes). Nas diversas respostas dos discentes, pelo menos uma dessas ações é destacada. Além de revelar o quanto essas práticas se repetem nas respostas, a análise dos questionários permitiu também perceber que os estudantes têm outras práticas para a preservação da saúde e a prevenção de doenças (momentos de lazer, práticas de atividades que promovam a felicidade, aproximação com a religião), como revelam as seguintes respostas:

Prática de atividades físicas regulares, a manutenção de alimentos saudáveis, meditação e o auto-questionamento, a realização de atividades que me tragam felicidade e satisfação.

\begin{abstract}
Pratico esportes, leio, mantenho uma alimentação saudável (sou vegetariano), mantenho comunhão diária com Deus, procuro, ao máximo, manter os meus relacionamentos.
\end{abstract}

Atividades físicas, alimentação balanceada, controle do stress cotidiano e tempo livre para o lazer.

Tais respostas reiteram que os participantes apresentam uma noção da complexidade do processo saúde-doença. Mesmo que a maioria dos alunos tenha demonstrado se importar com o cuidado à saúde e a prevenção de doenças, $8 \%$ (6 discentes) afirmaram não realizar nenhuma ação para manter a saúde ou prevenir doenças. Um deles respondeu: "Atualmente tenho negligenciado essas ações".

Além da prática de atividade física, visitas ao médico e alimentação saudável, dentre as respostas menos prevalentes alguns alunos destacaram práti- 
cas de prevenção como higiene, realização periódica de exames e, de forma mais tônica, uma preocupação com a vacinação, como pode ser percebido nos exemplos abaixo:

Alimentação saudável, prática de esportes, higiene pessoal básica, vacinação e exames regulares.

Faço sempre a higienização de tudo que vou consumir, cartão de vacinação em dias e sempre que necessário faço uso de métodos preventivos.

O estudo de Carvalho e Luz (2009) aponta a dificuldade de interpretação dos sentidos e significados culturais construídos sobre as práticas de saúde, ao considerar que estas se relacionam com os sentidos e valores culturais que atuam sobre o corpo, constituído por múltiplos aspectos que se realizam e se expressam nas relações sociais. Assim, as autoras analisam as práticas de saúde como um espaço de objetificação e articulação de elementos simbólicos, que podem apresentar grande mutabilidade, com planos e possibilidades variadas de articulação, que estão relacionadas também com a construção das concepções. A análise dos questionários permitiu perceber que as práticas de saúde adotadas são variada, portanto relacionada às escolhas e concepções de cada indivíduo.

Sobre as práticas utilizadas por eles, quando doentes, as respostas mais recorrentes foram: ir ao médico ou profissional de saúde (93\%) e prática de automedicação (71\%). Tais dados podem parecer controversos, pois se $93 \%$ dos entrevistados afirmam buscar um cuidado profissional quando doentes, o percentual de automedicação deveria ser menor. Entretanto, eles revelam uma simultaneidade dessas práticas: uma não exclui a outra.

A automedicação é conceituada por Naves e colaboradores (2010, p. 1752) como “[...] a seleção e uso de medicamentos para tratar doenças ou sintomas percebidos pelas pessoas sem a prescrição ou a supervisão de um médico ou dentista". A prática é usualmente a forma mais comum de resposta a sintomas. Embora seja necessário em uma função complementar aos sistemas de saúde, o hábito pode ter consequências indesejadas, como enfermidades ia- trogênicas e mascaramento de doenças evolutivas (AQUINO; BARROS; SILVA, 2010).

O estudo de Aquino, Barros e Silva (2010) acerca da automedicação entre acadêmicos de saúde aponta que esta prática é prevalente entre os discentes: $65,5 \%$ dos entrevistados confirmaram ter feito uso de medicamento na quinzena anterior; destes, $57,7 \%$ por meio de automedicação. As autoras consideram que esse percentual está dentro dos parâmetros encontrados na literatura, mas esperavam que o consumo de medicamentos fosse menor e mais racionalizado, por se tratarem de acadêmicos de saúde, que deveriam compreender o quão importante é a prescrição de remédio por um profissional especializado.

No estudo citado acima, não houve explicitação do semestre dos alunos entrevistados. Nesta pesquisa, os alunos do BI em Saúde são ingressantes no primeiro semestre do curso, com um percentual de automedicação tão elevado quanto. Isso demonstra que, para além do curso e do semestre em que estejam, temos que considerar a automedicação como um hábito cultural, acompanhado da facilidade em obter medicamentos sem prescrição.

0 elevado percentual de alunos que afirmam procurar um profissional de saúde quando doentes possibilita supor que eles têm o conhecimento acerca da importância da orientação profissional; no entanto, ainda assim praticam a automedicação. As respostas referentes ao uso de medicamentos reiteram isso, pois $64 \%$ dos participantes afirmaram utilizar medicamentos com prescrição, ao mesmo tempo em que $53 \%$ afirmaram utilizá-los sem prescrição. Dessa forma, os percentuais possibilitam considerar que os discentes fazem uso de remédios conforme ambas as formas: com e sem prescrição.

A automedicação tende a ocorrer especialmente quando os sintomas são mais comuns, o que pode estar relacionado com o alto percentual desta prática. Ainda se pode pensar que o elevado uso de remédio com prescrição pode estar relacionado com o insucesso da automedicação. É importante também considerar que o gênero pode ser um determinante para a prática da automedicação, tendo em vista que 
os homens buscam menos os serviços de saúde que as mulheres, como apontado na Política Nacional de Atenção Integral à Saúde do Homem (BRASIL, 2009).

Em relação às práticas de saúde alternativas e/ou complementares, $32 \%$ dos entrevistados afirmaram utilizá-las. Dentre essas, as que obtiveram maior destaque foram: massagem (20\%), homeopatia (14\%), fitoterapia (13\%), banho de folhas (10\%) e RPG (10\%). Mesmo que a utilização das práticas alternativas e integrativas apareça de forma moderada entre os discentes, os dados corroboram com a Política Nacional de Práticas Integrativas e Complementares (PNPIC), apontando que há uma crescente legitimação social dessas práticas (BRASIL, 2006). Otani e Barros (2011), em artigo de revisão de literatura, também consideram que existe um interesse crescente por parte de usuários, profissionais e gestores nos conceitos de Medicina Integrativa e Medicinas Alternativas e Complementares, de modo que são necessárias a implementação e o gerenciamento de novas práticas de cuidado e cura.

Ayres (2001), ao discutir (inter)subjetividades, aponta que a importância do controle da doença não deve ser diminuída. No entanto, “deve ser revista sua exclusividade como critério normativo de sucesso das práticas de saúde" (AYRES, 2001, p. 7). Como mencionado, o autor considera que as (inter)subjetividades são caracterizadas pela mobilidade do sucesso prático. Nessa perspectiva, quando o sujeito opta por práticas de saúde alternativas, a sua escolha deve ser respeitada, haja vista que as escolhas das práticas de saúde estão relacionadas com o caráter relacional das identidades e historicidades dos indivíduos e grupos. Nesse sentido, a PNPIC aponta que a adoção das práticas de saúde alternativas está relacionada com os diferentes processos de adoecimento e de saúde, de forma que o indivíduo deve ser considerado de modo global (BRASIL, 2006).

Sobre as práticas adotadas quando se está doente, além das práticas alternativas e complementares, houve destaque também para a realização de tratamentos religiosos (20\% entre os entrevistados). Tais práticas se relacionam com a complexidade do processo do adoecimento e do caráter relacional das es- colhas, tendo em vista que a adoção de uma prática religiosa está relacionada com aspectos culturais e subjetivos. A adoção de práticas religiosas é, também, uma forma de enfrentamento da doença, de forma que a fé não está dissociada do processo de adoecimento (MURAKAMI; CAMPOS, 2012). Nesse sentido, Murakami e Campos (2012), ao estudarem a relação da saúde com a religiosidade, apontam que a religião é um elemento que faz parte da subjetividade, capaz de atribuir significado ao sofrimento.

A análise das respostas dos discentes do BIS/ UFBA permite perceber que há uma preocupação com a própria saúde e uma percepção de sua complexidade. As práticas de saúde predominantes representam um desvio do padrão, já que destoam do que vem sendo apontado na literatura, de que o padrão de masculinidade pode trazer déficit para a saúde (CONNELL, 1995; FIGUEIREDO, 2005).

\section{CONCLUSÃO}

Entre os discentes participantes deste estudo, o binômio saúde-doença é percebido como fenômeno complexo, que envolve diversos fatores. Em relação às práticas adotadas, nota-se que é majoritária a preocupação com hábitos preventivos e hábitos de vida saudável. É interessante perceber que a grande maioria dos estudantes busca realizar alguma ação para manter a saúde ou prevenir doenças; a proporção de alunos que não realizam nenhuma dessas ações é muito baixa.

As respostas mais prevalentes entre os discentes estão relacionadas à ideia de saúde como bem-estar, conforme o conceito de saúde da OMS. Foi possível perceber que, atrelado à noção de bem-estar individual, eles se referiram à qualidade de vida em seu âmbito coletivo, envolvendo entretenimento, cultura e educação. Esses aspectos se distanciam das concepções e práticas ligadas à saúde entre aqueles que reproduzem o modelo hegemônico de masculinidade, como vimos. Nessa perspectiva, as práticas de saúde dos estudantes estão ligadas majoritariamente à alimentação saudável e à atividade física, bem como à 
automedicação e à busca por profissionais de saúde, quando doentes. Práticas alternativas e religiosas ligadas à saúde foram poucas vezes mencionadas, mas refletem a multiplicidade dos saberes e racionalidades envolvidas nesse processo.

Como mencionado, muitos estudos apontam a existência de uma masculinidade hegemônica que seria prejudicial à saúde dos homens, associada às noções de invulnerabilidade, comportamento de risco e pouca preocupação com a própria saúde. No entanto, esta pesquisa e outros estudos acerca das representações sociais da saúde, entre os homens, apontam uma tendência à mudança, na medida em que os homens têm demonstrado maior preocupação com o complexo processo saúde-doença.

Nessa direção, destaca-se a necessidade de novas pesquisas - com incentivo social, midiático e das universidades - que abordem a saúde e a desconstrução dos modelos de masculinidade que produzem um distanciamento dos homens em relação à mesma, de modo a ampliar as reflexões realizadas por este e outros estudos, abordando as suas relações com diversos fatores psicossocioculturais.

\section{REFERÊNCIAS}

AQUINO, D.S.; BARROS, J.A.C.; SILVA, M.D.P. A automedicação e os acadêmicos da área de saúde. Ciência \& Saúde Coletiva [on-line], Rio de Janeiro, v.15, n.5, p.2533-2538, 2010. Disponível em: <http:// goo.gl/CBaP1B>. Acesso em: 2 maio 2016.

AYRES, J.R.C.M. Sujeito, intersubjetividade e práticas de saúde. Ciência \& Saúde Coletiva [on-line], Rio de Janeiro, v.6, n.1, p.63-72, 2001. Disponível em: <http://goo.gl/MmDxFs>. Acesso em: 2 maio 2016.

BARDIN, L. Análise de conteúdo. 4.ed. rev. e actual. Lisboa, PT: 70, 2009.

BATISTELLA, C. Abordagens contemporâneas do conceito de saúde. In: Fonseca, A.F. (Org.).
0 território e o processo saúde-doença. Rio de Janeiro: EPSJV, FIOCRUZ, 2007. p.51-86.

\section{BRASIL, MINISTÉRIO DA SAÚDE. Secretaria de} Vigilância em Saúde. Política Nacional de Atenção Integral à Saúde do Homem. Brasília: Ministério da Saúde, 2009.

\section{BRASIL. MINISTÉRIO DA SAÚDE. Política Nacional de Práticas Integrativas e Complementares no SUS. Brasília: Ministério da Saúde, 2006.}

BORUCHOVITCH, E.; FELIX-SOUSA, I.C.; SCHALL, V.T. Conceito de doença e preservação da saúde de população de professores e escolares de Primeiro Grau. Rev. Saúde Pública, São Paulo, v.25, n.6, p.418-425, 1991. Disponível em: <https://goo.gl/M2xSVa>. Acesso em: 16 fev. 2017.

BUSS, P.M.; PELLEGRINI FILHO II, A. A saúde e seus determinantes sociais. Physis, Rio de Janeiro, v.17, n.1, p.77-93, 2007. Disponível em: <http://goo.gl/ sTWTcS>. Acesso em: 16 fev. 2017.

CAMARGO, B.V.; et al. Representações sociais de saúde e cuidado: um estudo multicêntrico sobre vulnerabilidade masculina. Temas em Psicologia, Ribeirão Preto, v.19, n.1, p.179-192, jun. 2011. Disponível em: <http://goo.gl/MyGgbM>. Acesso em: 8 jun. 2016.

CARVALHO, M.C.V.S.; LUZ, M.T. Práticas de saúde, sentidos e significados construídos: instrumentos teóricos para sua interpretação. Interface (Botucatu) [on-line], v.13, n.29, p.313326, 2009. Disponível em: <http://goo.gl/BDCRmE>. Acesso em: 2 maio 2016.

CONNELL, R. Masculinities: knowledge, power and social change. Berkeley, Los Angeles: University of California Press, 1995.

CZERESNIA, D. O conceito de saúde e a diferença 
entre prevenção e promoção. In: CZERESNIA, D.; FREITAS, C. (Org.). Promoção da saúde: conceitos, reflexões, tendências. Rio de Janeiro: Fiocruz, 2009. p.19-43.

FIGUEIREDO, W. Assistência à saúde dos homens: um desafio para os serviços de atenção primária. Ciênc. Saúde Coletiva [on-line], v.10, n.1, p.105-109, 2005. Disponível em: <http://goo.gl/5MkG17>. Acesso em: 22 mar. 2016.

GOMES, R. A saúde do homem em foco. São Paulo: UNESP, 2010.

MURAKAMI, R.; CAMPOS, C. Religião e saúde mental: desafio de integrar a religiosidade ao cuidado com o paciente. Revista Brasileira de Enfermagem [online], Brasília, v.65, n.2, p.361-367, 2012. Disponível em: <http://goo.gl/ke7V2V>. Acesso em: 9 maio 2016.

NASCIMENTO, A.R.A.; et al. Masculinidades e práticas de saúde na região metropolitana de Belo Horizonte - MG. Saúde e Sociedade [on-line], São Paulo, v.20, n.1, p.182-194, 2011. Disponível em: <http://goo.gl/j1nZx0>. Acesso em: 2 maio 2016.

NAVES, J. O. S.; et al. Automedicação: uma abordagem qualitativa de suas motivações. Ciência \& Saúde Coletiva [on-line], Rio de Janeiro, v.15, n.1, p.1751-1762, 2010. Disponível em: <http://goo.gl/ khV8xq>. Acesso em: 2 maio 2016.
OTANI, M.A.P.; BARROS, N.F. A Medicina Integrativa e a construção de um novo modelo na saúde. Ciência \& Saúde Coletiva [on-line], Rio de Janeiro, v.16, n.3, p.1801-1811, 2011. Disponível em: <http://goo.gl/ RQ1gnw>. Acesso em: 2 maio 2016.

SCHRAIBER, L.B.; GOMES, R.; COUTO, M.T. Homens e saúde na pauta da Saúde Coletiva. Ciência \& Saúde Coletiva [on-line], Rio de Janeiro, v.10, n.1, p.717, 2005. Disponível em: <http://goo.gl/nn92m7>. Acesso em: 2 maio 2016.

SCLIAR, Moacyr. História do conceito de saúde. Physis [on-line], Rio de Janeiro, v.17, n.1, p.29-41, 2007. Disponível em: <http://goo.gl/eVxti3>. Acesso em: 28 mar. 2016.

SILVA, B.F. Processo saúde-doença: representações sociais de homens assistidos pelo Programa Saúde da Família. 2013.Dissertação (Mestrado) Universidade Federal de Juiz de Fora, Juiz de Fora, 2013. Disponível em: <http://goo.gl/57pzPE>. Acesso em: 8 jun. 2016.

SILVA, I.J. et al. Cuidado, autocuidado e cuidado de si: uma compreensão paradigmática para o cuidado de enfermagem. Rev. esc. enferm. USP, São Paulo, v.43, n.3, p.697-703, 2009. Disponível em: <http://goo.gl/REN3nK>. Acesso em: 30 mar. 2016.

TEIXEIRA, C.F.; VILASBOAS, A.L.Q. Modelos de atenção à saúde no SUS: transformação, mudança ou conservação? In: PAIM, J.S.; ALMEIDA FILHO, N. Saúde Coletiva: teoria e prática. São Paulo: MedBook, 2013. p.287-301. 
1 Professora Permanente do Programa de Pós-Graduação em Estudos Interdisciplinares Sobre a Universidade (PPGEISU) da Universidade Federal da Bahia; Doutora em Saúde Pública, psicóloga e Coordenadora do Núcleo de Estudos Interdisciplinares em Saúde, Violência e Subjetividade - SAVIS. E-mail: therezacoelho@gmail.com

2 Mestranda do Programa de Pós-Graduação em Estudos Interdisciplinares Sobre a Universidade - PPGEISU da Universidade Federal da Bahia, Bacharela em Saúde e graduanda do Bacharelado Interdisciplinar em Humanidades pela Universidade Federal da Bahia. E-mail: danielep.rocha@hotmail.com

Avaliado em: 7 de fevereiro de 2017

Aceito em : 12 de março de 2017

3 Graduanda do Bacharelado Interdisciplinar em Saúde pela Universidade Federal da Bahia. E-mail: souraquelaraujo@gmail.com 